\title{
Long-Distance Time Transfer in Optical Fiber Networks Using a Cascaded Taming Technology
}

\author{
Ding Chen $\mathbb{D}^{1,2}$, Jiangning Xu $\mathbb{D},{ }^{1}$ Yifeng Liang $\mathbb{D}^{1},{ }^{1}$ Shan Jiang, ${ }^{3}$ and Hongyang $H e \mathbb{D}^{1}$ \\ ${ }^{1}$ Department of Navigation Engineering, PLA Naval University of Engineering, Wuhan 430033, China \\ ${ }^{2}$ College of Electronic Engineering, Jiujiang University, Jiujiang 332005, China \\ ${ }^{3}$ Admin Office, PLA Naval University of Engineering, Wuhan 430033, Hubei, China \\ Correspondence should be addressed to Hongyang He; xgdhehongyang@163.com
}

Received 15 September 2020; Revised 4 April 2021; Accepted 9 April 2021; Published 22 April 2021

Academic Editor: Bosheng Song

Copyright (C) 2021 Ding Chen et al. This is an open access article distributed under the Creative Commons Attribution License, which permits unrestricted use, distribution, and reproduction in any medium, provided the original work is properly cited.

In order to meet the time service needs of high-precision, long-distance, and multinode optical network, this paper proposes a new time synchronization solution, which combines the wavelength division multiplexing (WDM) technology with cascaded taming clock technology. The WDM technology is used for time synchronization between each pair of master-slave nodes. In the system, there are two wavelengths on the fiber link between the master node and the slave node for transmitting signals. 1 plus per second (PPS) signal, time code signal, and $10 \mathrm{MHz}$ signal are, respectively, and successively, sent to the optical fiber link. By solving the one-way delay through analysis of error contribution and link characteristics of the time transmission process, time synchronization of the master-slave nodes pair is achieved. Furthermore, the authors adopt cascaded taming clock technology to ensure accurate time synchronization of each node. A $700 \mathrm{~km}$ long-distance time-frequency synchronization system is constructed in the laboratory. The system uses a cesium atomic clock as the reference clock source and transmits the signals through 8 small rubidium atomic clocks (RB clocks) hierarchically. Results from the experiment show that the long-term time stability is $47.5 \mathrm{ps} / 10^{4} \mathrm{~s}$. The system's structural characteristics and the experiment results meet the requirements to allow practical use of high-precision time synchronization in networks. This proposed solution can be applied in various civil, commercial, and military fields.

\section{Introduction}

Time is an essential fundamental physical quantity, which has become the physical quantity with the highest measurement accuracy among the seven international basic units [1-3]. Time synchronization technology refers to the transmission of a standard time signal from source to terminal employing transmission medium. Through time comparison, the system deviation between clock sources is eliminated, and the time signal with high stability and high precision is provided to the remote time users [4-6]. There are two reasons to promote the development and improvement of time synchronization technology. On one hand, with the continuous breakthrough of time-keeping accuracy, the current clock source with the highest precision has achieved $10^{-16}$ second stability and $10^{-18}$ day stability, respectively $[7,8]$. The improvement of clock performance puts forward higher requirements for the accuracy of time synchronization. On the other hand, scientific research, navigation and positioning, aerospace, power transmission, military security, and other fields are continually improving the demand for time synchronization accuracy and stability $[9,10]$. Therefore, high-precision time synchronization has become an important topic in the current scientific research.

In the existing time-frequency synchronization scheme, satellite timing has become the primary way with the advantages of a wide range and flexible access, but it has obvious security risks because of the weak signal, which is easy to interfere with. For the sake of national defense security, the US Department of Defense began to plan the construction of national integrated Positioning, Navigation, and Timing (PNT) in 2010 [10-12]. It would develop various 
timing methods for cooperation to make up for the defects of any single system and make PNT more perfect $[13,14]$. The construction of China's PNT system has also entered an accelerated stage since 2015. In October 2019, the China National Time Service Center (NTSC) announced that it planned to build a three-dimensional cross time service system in five years. In the future time service system, optical fiber, satellite, longwave, and other ways complement each other to realize mutual backup between heaven and Earth [15]. In this background, with the advantages of high precision, high reliability, and low loss, optical fiber has become one of the main directions of time-frequency synchronization technology [16].

Time-frequency information transmission by optical fiber began in the 1970s. So far, many research institutions have verified the optical fiber time-frequency synchronization scheme with excellent accuracy. For example, Smotlacha et al. used $744 \mathrm{~km}$ WDM optical fiber transmission experiment to achieve the time stability of $8.17 \mathrm{ps} /$ $500 \mathrm{~s}$ in 2010 [17]. Sliwczynski et al. adopted the round-trip method to achieve a synchronization accuracy of $50 \mathrm{ps}$ in the $615 \mathrm{~km}$ WDM optical fiber time synchronization experiment in 2015 [18]. Wu Guiling et al. adopted the BTDM scheme in the $2000 \mathrm{~km}$ optical fiber experiment, which achieved a time transfer stability of $23 \mathrm{ps} / 10^{5} \mathrm{~s}$ and a time synchronization accuracy of $30 \mathrm{ps}[19,20]$.

All the above researches have achieved excellent transmission accuracy and stability. But most of these schemes used point-to-point time transmission in longdistance optical signal transmission, in which the optical amplifier was used to extend the transmission length. The distributed network and multipoint download of optical fiber time transfer have not to be considered enough. With the increasing demand for time-frequency distribution for multiple users in the same area, the optical fiber timefrequency synchronization scheme needs to meet the needs of distributed, multistations, and different network topology.

Based on the above requirements, this paper proposes a new time synchronization scheme that combines WDM time transfer technology and cascaded taming clock technology. In this system, an RB clock is placed every $100 \mathrm{~km}$. The time comparison between the adjacent $\mathrm{RB}$ clocks is carried out by the round-trip method, and the clock taming module tames the slave clock. It can transmit the timefrequency signal to link length less than 100 kilometers without an optical amplifier between any two adjacent nodes. Besides, each node can be used as a clock source to connect multiple nodes and has independent time-keeping and timing functions. This feature can better meet the requirements of distributed networking and multisite download of the optical fiber network. The scheme has the advantages of high transmission accuracy, high redundancy, high security, and flexible networking. According to statistics, about $80 \%$ of 239 cities in 24 provinces in eastern China are less than $100 \mathrm{~km}$ away from neighboring cities. Commercial optical cables have been laid along highways and railways between these cities. Therefore, this scheme is extremely suitable for the realization of high-precision time- frequency synchronization between cities and meets the needs of time synchronization practicality and networking in many fields.

\section{Principle and System Configuration}

2.1. Time Transfer Principle. In this system, WDM technology is used for time bidirectional transmission, which can ensure that the physical length of the round-trip link is entirely symmetrical. A $100 \mathrm{~km}$ optical fiber link is connected between the master and slave nodes, on which the time-frequency signal is transmitted by microwave. The twoway time transfer process between the master node and the slave node is shown in Figure 1.

$\mathrm{E} / \mathrm{O}$ is an electrooptical converter, which converts the modulated electrical signal into optical signal for optical fiber transmission in the master node. OS is an optical transmitting device, which transmits optical signals to the optical fiber link. OR is an optical receiving device, which receives the optical signal from the optical fiber link in the slave node. $\mathrm{O} / E$ is the electrooptical converter, which converts the received optical signal into electrical signal and sends it to the demodulation equipment in the slave node.

The time synchronization steps from the master node to the slave node are described below in detail. In the same way, the time synchronization between other master-slave nodes is the same.

(1) $\mathrm{Rb}$ clock 1 of the master node receives time-frequency information from the superior clock source. Then master node outputs 1 PPS signal, $10 \mathrm{MHz}$ frequency signal, and time of day (TOD) signal to the slave node.

(2) The output signal of Rb clock 1 is divided into two channels; one channel is sent to time-to-digital conversion (TDC) to start timing, and the other channel is modulated and encoded by Modulate (MOD) to become a signal suitable for optical module transmission.

(3) After being processed by MOD, the electrical signal is sent to the optical module and becomes an optical signal. Then, the optical signal is transmitted to the optical fiber link at wavelength $\lambda_{1}$.

(4) The optical signal is transmitted to the slave node through the optical fiber link. After being filtered, the optical signal is transmitted to the optical module for photoelectric conversion and then decoded by Demodulate (DEM). Through the synchronization of position and frame number, the time-frequency information is recovered. Finally, it is converted into an optical signal and transmitted back to the master node in wavelength $\lambda_{2}$.

(5) After the optical signal is returned from the slave node to the master node, the optical signal is photoelectrically converted in the optical module. The TDC stops counting when the signal is detected. From the TDC measurement results, the total roundtrip delay of one cycle can be obtained. 


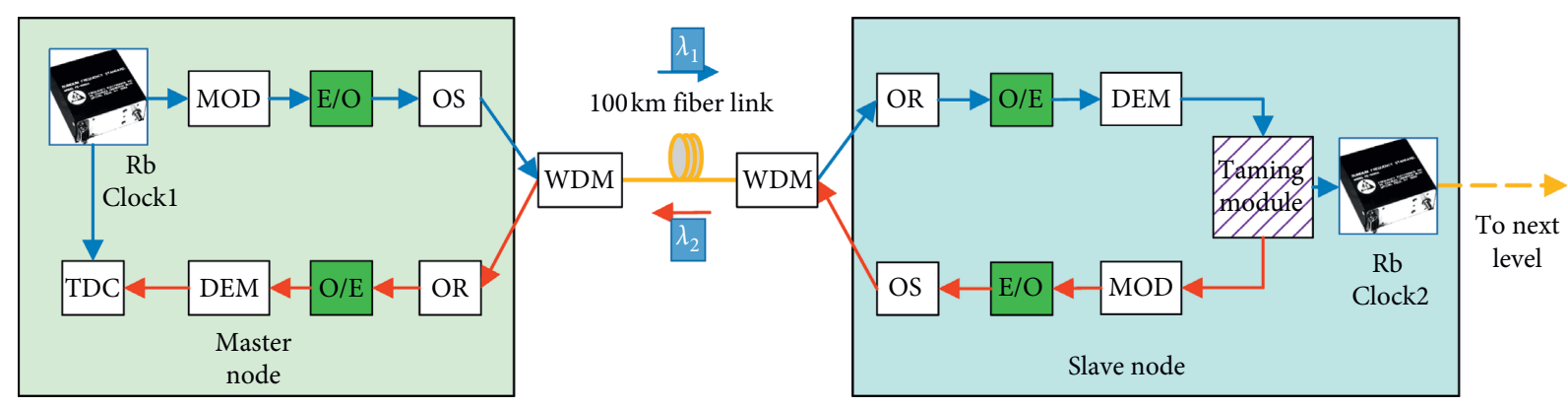

FIGURE 1: Block diagram of bidirectional transfer system.

(6) According to the wavelengths $\lambda_{1}$ and $\lambda_{2}$, and the fiber length, the round-trip delay difference in the fiber link is calculated. TDC can measure the sum of the round-trip delay. The high-precision instrument can measure transmitting and receiving a delay of each module. According to the above data, the one-way transmission delay from the master node to the slave node can be calculated.

(7) According to the one-way transmission delay from the master node to the slave node, 1 PPS is precompensated in the master node. The compensated time-frequency information is sent to the slave node. The 1 PPS time signal and $10 \mathrm{MHz}$ frequency signal generated in the slave node are used as the reference signal to tame RB clock 2, to realize master-slave phase locking.

(8) After the time synchronization with the master node is completed, the slave node transfers the corrected time-frequency signal to the next level node, which becomes the master node of the next level node.

In this system, G.652 optical fiber is used. The back and forth wavelengths are $1490 \mathrm{~nm}$ and $1550 \mathrm{~nm}$, respectively. At present, 1490-1550 nm single fiber bidirectional wavelength division multiplexing module is widely used with low cost, so $1490-1550 \mathrm{~nm}$ wavelengths are more conducive to engineering application. Using the round-trip delay ratio method in this paper, assuming that the daily temperature fluctuation of $100 \mathrm{~km}$ fiber core is $1^{\circ} \mathrm{C}$, using different wavelength intervals has little effect on the accuracy of time synchronization. Considering the existing experimental conditions, system cost control, engineering requirements, and nanosecond target accuracy, we choose the wavelength combination of $1490-1550 \mathrm{~nm}$.

TDC is a small time interval measurement device, which is used to measure the round-trip delay. The 1 PPS transfer sequence diagram of the single transfer process is shown in Figure 2.

In Figure 2, the time delay values of time transfer between master and slave nodes can be expressed by (1) and (2):

$$
\begin{aligned}
& \tau_{m s}=\tau_{m t}+\tau_{f m s}+\tau_{s r}, \\
& \tau_{s m}=\tau_{s t}+\tau_{f s m}+\tau_{m r},
\end{aligned}
$$

where $\tau_{m s}$ is the transmission delay from the master node to the slave node, that is, the delay of time signal transmission from RB clock 1 to RB clock 2. $\tau_{s m}$ is the transmission delay from the master node to the slave node, that is, the time delay of time signal from RB clock 2 back to RB clock $1 . \tau_{m t}\left(\tau_{s t}\right)$ is the delay of the transmitting circuit of the master node (slave node). $\tau_{m r}\left(\tau_{s r}\right)$ is the delay of the receiving circuit of the master node (slave node). $\tau_{f m s}$ is the transmission delay of the signal from the master node to the slave node in the optical fiber link; $\tau_{f s m}$ is the transmission delay of the signal from the slave node to the master node in the optical fiber link.

By adding (1) and (2), the total round-trip transmission delay $\tau$ of the master and slave nodes can be obtained.

$$
\tau=\tau_{m s}+\tau_{s m}=\tau_{m t}+\tau_{f m s}+\tau_{s r}+\tau_{s t}+\tau_{f s m}+\tau_{m r} .
$$

In (3), $\tau$ is the total round-trip delay sum of the loopback period of a master-slave node, which can be measured by the TDC of the master node. TDC is installed in the master node to measure the round-trip delay sum. When the master node sends a signal to the slave node at $T_{\mathrm{A}}$, TDC starts to count. After the signal is looped back from the slave node, when it reaches the master node at $T_{\mathrm{C}}$, TDC stops counting. TDC measurement results can get the total round-trip delay of the last cycle $\left(\tau=T_{C}-T_{A}\right) \cdot \tau_{m t}, \tau_{s r}, \tau_{s t}$ and $\tau_{m r}$ can be measured in advance before the experiment. Their positions are on the master-slave nodes, and the environment temperature is easy to be controlled under constant temperature. Therefore, these hardware delays are extremely stable and can be used as fixed values.

Equation (4) can be obtained from (3). Since the right of (4) is determined, the value of $\tau_{f m s}+\tau_{f s m}$ can be obtained:

$$
\tau_{f m s}+\tau_{f s m}=\tau-\left(\tau_{m t}+\tau_{s r}+\tau_{s t}+\tau_{m r}\right)
$$

The ratio of transmission delay of round-trip optical signal with different wavelengths of the same optical fiber can be obtained:

$$
\rho=\frac{\tau_{f s m}}{\tau_{f m s}}=\frac{n_{f s m}-\lambda_{f s m} d n_{f s m} / d \lambda_{f s m}}{n_{f m s}-\lambda_{f m s} d n_{f m s} / d \lambda_{f m s}} .
$$

In (5), $n$ is the refractive index, and $\lambda$ is the wavelength of the optical signal. The values of $\left(\tau_{f m s}+\tau_{f s m}\right)$ and $\left(\tau_{f m s} / \tau_{f s m}\right)$ can be obtained. According to (1), (2), (3), (4), 


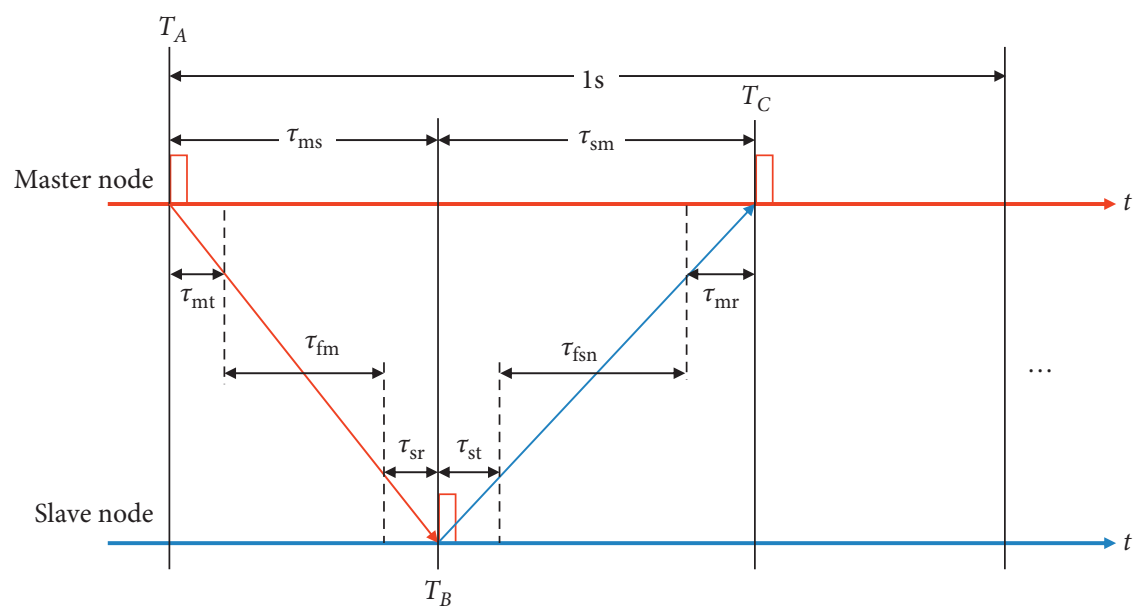

FIgURE 2: Time sequence diagram of the bidirectional transfer system.

and (5), the calculation equation of transmission delay $\tau_{m s}$ from master node to slave node can be obtained.

$$
\tau_{m s}=\frac{\rho\left(\tau-\tau_{m r}-\tau_{s t}\right)+\left(\tau_{m t}+\tau_{s r}\right)}{\rho+1},
$$

$\tau$ can be measured by TDC, $\rho$ can be calculated by (5) $\tau_{m t}, \tau_{s r}$, $\tau_{s t}$ and $\tau_{m r}$ can be premeasured. Finally, the calculation of $\tau_{m s}$ is completed at the slave node.

The average value of $\tau_{m s}$ is calculated by multiple measurements to reduce the error. The delay compensation module compensates the time signal of the slave node, and the standard 1 PPS signal is recovered finally.

2.2. Clock Taming Principle. The process of clock taming is as follows: first, compare the 1 PPS output by dividing the clock signal frequency from the slave node with the 1 PPS that has been regenerated and has passed the delay compensation to get the time difference value. Then, the instantaneous deviation between the slave frequency and the standard frequency is calculated. Finally, the frequency of the slave atomic clock is calibrated according to the voltage control value obtained from the conversion of deviation value. At the same time, the phase of the slave clock needs to be compensated according to the time difference value, to keep the rising edge of the master-slave clock pulse consistent and achieve "phase lock" through continuous frequency comparison.

The design of the clock taming module in this paper is independent of the optical fiber time transfer system. Clock taming is carried out after the master node reference timefrequency information is regenerated. The reference information and the time-frequency information output by the acclimation module are processed to obtain the phase difference. The control module adjusts the phase difference roughly and finely. Then, the updated time-frequency information and reference information are repeatedly compared and adjusted. The basic structure of the acclimation system is shown in Figure 3(a):
The functions of the main modules of the clock taming system are as follows:

(1) Phase detector: It is a digital phase detector based on FPGA, whose primary function is to identify the phase relationship between the slave node and the reference information. When the relative frequency difference of the master-slave clock is small, the phase discriminator starts to compare the slave information with the reference information and sends the judged leading or lagging state to the filtering and gain adjusting module.

(2) Filter processing and gain adjustment: Process the phase discrimination data. When the relative clock difference between the slave node and master node is obtained, the module generates the control quantity of digital to analog conversion. By adjusting the loop filter gain, the master-slave clock drift can be filtered out.

(3) High-precision digital to analog conversion module: The high-resolution digital to analog converter adjusts the slave clock in small steps $\left(2.56 \times 10^{-7} \mathrm{~V}\right)$ and large dynamic range $(0-5 \mathrm{~V})$ to ensure that the slave clock can be adjusted accurately.

(4) Frequency divider: It divides the output signal of the slave node to meet the requirements of comparison with the reference information. For example, in this system, the reference signal of the master node is $1 \mathrm{~Hz}$, so the frequency signal of $1 \mathrm{~Hz}$ is generated in the frequency divider of the slave node.

As shown in Figure 3(b), the physical board card of the acclimation system can be used in a $1 \mathrm{U}$ high terminal computer, which includes a rubidium atomic clock, phase detector, and digital to analog conversion module.

2.3. System Configuration. Based on the time transfer technology and clock taming technology described above, we built a $700 \mathrm{~km}$ optical fiber transmission system in the laboratory. The system includes 8 nodes connected linearly. 


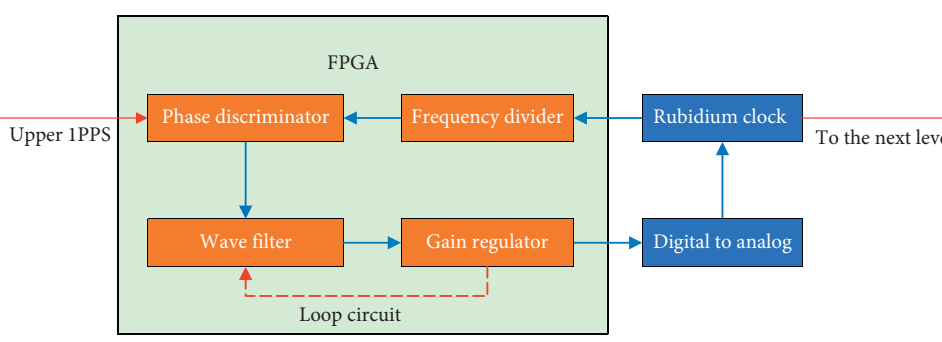

(a)

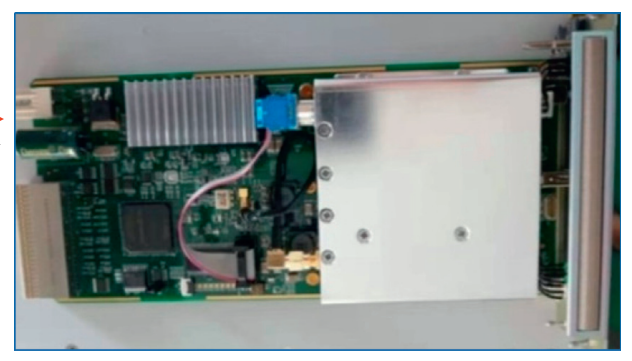

(b)

Figure 3: Rubidium atomic clock taming system. (a) Block diagram. (b) Physical diagram.

The adjacent nodes are connected by $100 \mathrm{~km}$ optical fiber of G.652. Each node equipment is placed in a $1 \mathrm{U}$ terminal machine, including a rubidium atomic clock, transceiver, and taming module. The configuration chart of the $700 \mathrm{~km}$ optical fiber time transfer experiment is shown in Figure 4.

Among them, the rubidium clock of the first node takes the TOD of the satellite as the initial time information and receives the $1 \mathrm{PPS}$ and $10 \mathrm{MHz}$ of the cesium atomic clock as the reference time-frequency information. After building the system, the time interval counter SR620 is connected between node 1 and node 8 to measure the time synchronization result of the system. The rubidium atomic clock of node 2 -node 7 is not only the slave clock of the previous node, but also the master clock of the next node.

\section{Experimental Results}

At present, there is no uniform time length regulation for the long-term and short-term performance of optical fiber timefrequency synchronization. According to the test records of literature [17], literature [19], and others, more than 10-hour test is enough to verify the short-term performance of the system, and more than 48-hour test can prove the long-term performance of the system.

3.1. Short-Term Performance Tests. Due to some unforeseen factors in the short-term performance test, to make the performance test more convincing, this paper has carried out four independent experiments and collected four periods of data about 14-15 hours. During the whole experiment, the working temperature of the terminal equipment was controlled in a small fluctuation by air conditioning. The experimental steps and results are as follows:

(1) $700 \mathrm{~km}$ optical fiber time-frequency synchronization system is connected step by step according to Figure 4.

(2) The rubidium atomic clock is turned on. The equipment at all nodes is initialized for 1 hour (the data does not need to be recorded).

(3) Node 1 rubidium atomic clock and node 8 rubidium atomic clock are, respectively, connected with SR620 through $2 \mathrm{~m}$ customized optical fiber. The phase values are compared in the counter, and the data are observed and recorded in real-time.
(4) The experiment was carried out for 14-15 hours, and the data was recorded, then the counting was stopped, the equipment was shut down, and each node was disconnected.

(5) Repeat (1)-(4) processes once a day to collect four groups of data.

Four groups of clock difference data obtained from short-term performance test of optical fiber time-frequency synchronization system are shown in Figure 5. The standard deviation (SDEV) calculated from the original clock difference data is shown in Table 1 .

The TDEV of four groups of experiments is shown in Figure 6. The statistics of TDEV data are shown in Table 2.

The Allan deviation (ADEV) obtained from four groups of experiments is shown in Figure 7. The statistics of ADEV data are shown in Table 3.

By analyzing the above four independent test results, the time delay generated by the types of equipment can cancel each other, as long as the temperature of the laboratory is relatively stable. Finally, the SDEV of all tests is less than 500 ps, the TDEV of the second scale and 10000-second scale is less than $100 \mathrm{ps}$, and the frequency stability is in the order of $10^{-14} / 10^{4} \mathrm{~s}$.

3.2. Long-Term Performance Tests. From 23:00 on August 13 to $14: 30$ on August 16, 2019, 63.5 hours of experimental data was collected, in which 8 hours of temperature change was added at 06:00 on August 16. The temperature box used is CTP404, and its temperature range is $-40^{\circ} \mathrm{C}$ to $100^{\circ} \mathrm{C}$. For buried or suspended optical fiber, the temperature change of $-20^{\circ} \mathrm{C}$ to $40^{\circ} \mathrm{C}$ can cover the general situation. The temperature range of this experiment is $-20^{\circ} \mathrm{C}$ to $40^{\circ} \mathrm{C}$ because the optical fiber disk is used instead of the optical fiber cable. The experimental steps are as follows:

(1) $700 \mathrm{~km}$ optical fiber time-frequency synchronization system is connected step by step according to Figure 4 , in which the optical fiber disk of the $600-650 \mathrm{~km}$ section is placed in the temperature box.

(2) The rubidium atomic clock is turned on. The equipment at all nodes is initialized for 1 hour (the data does not need to be recorded).

(3) Node 1 rubidium atomic clock and node 8 rubidium atomic clock are, respectively, connected with SR620 


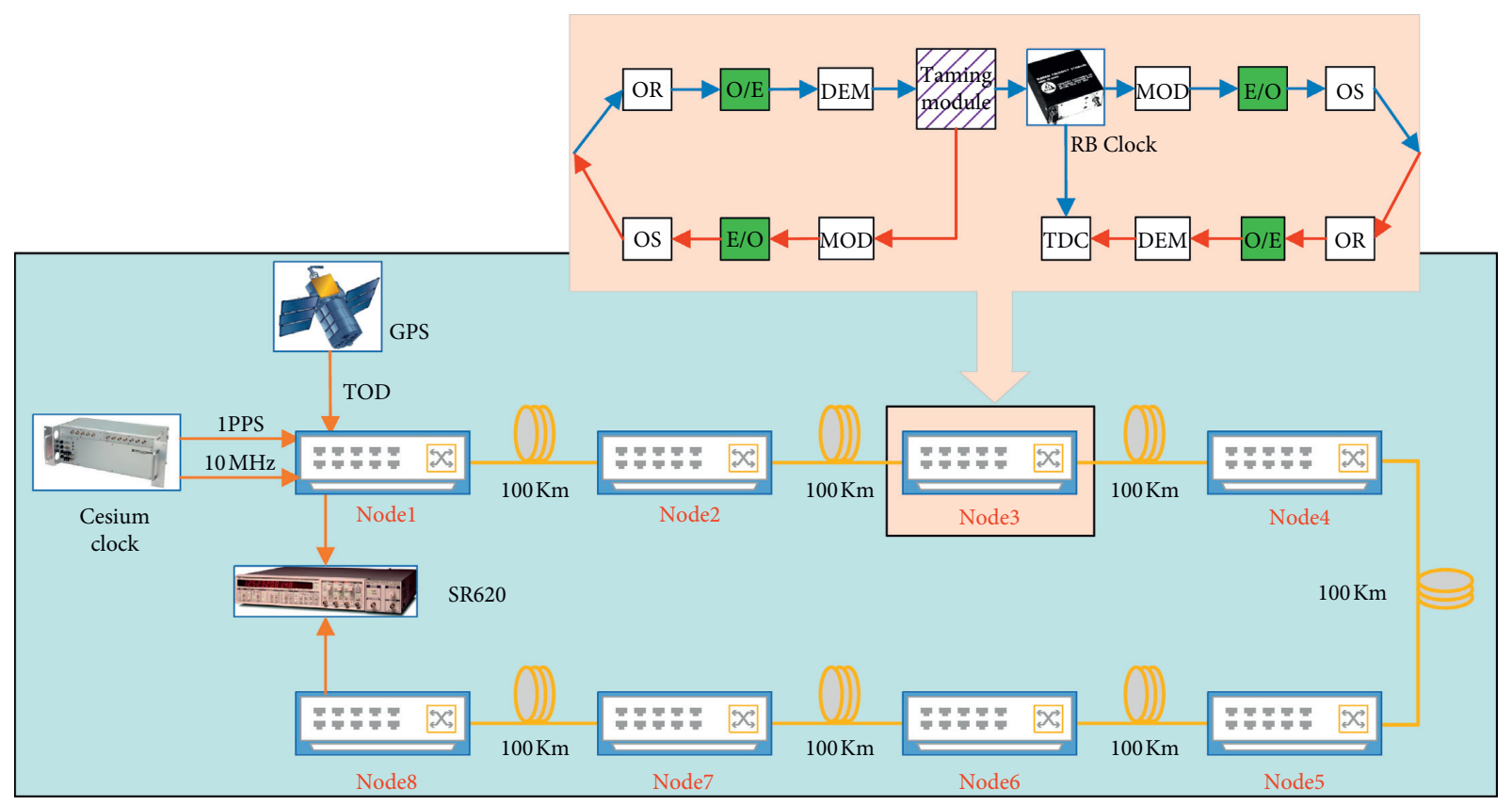

FIgURE 4: Configuration chart of $700 \mathrm{~km}$ optical fiber time transfer experiment.

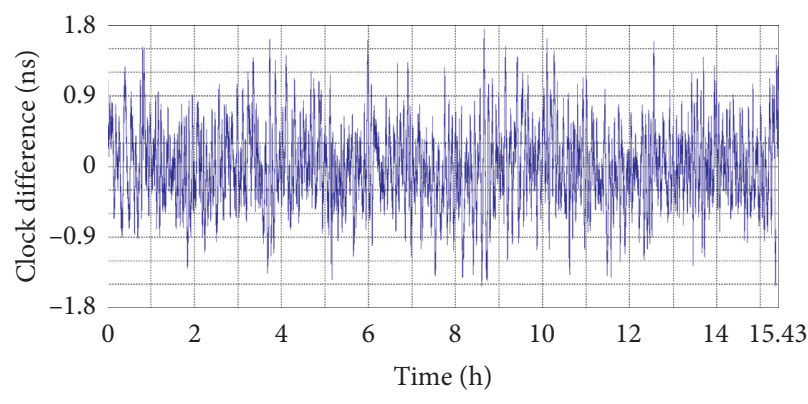

Group 1
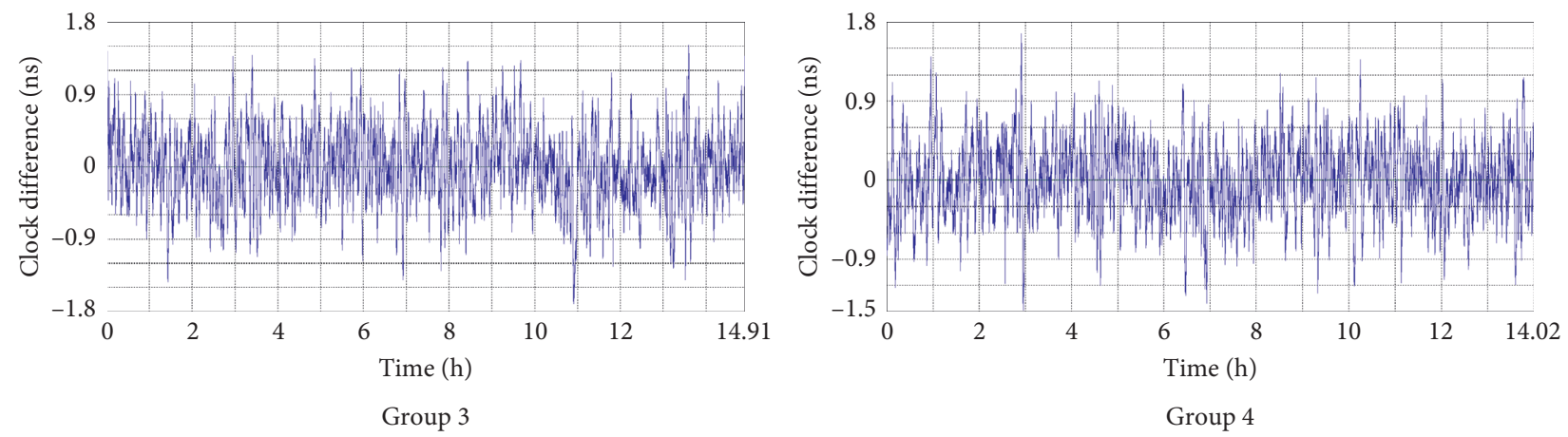

FIgURE 5: Clock difference at two ends of $700 \mathrm{~km}$ optical fiber. (a) Group 1. (b) Group 2. (c) Group 3. (d) Group 4.

TABLE 1: Sdev of four short-term performance tests.

\begin{tabular}{lccc}
\hline Group & Duration $(\mathrm{h})$ & Temperature $\left({ }^{\circ} \mathrm{C}\right)$ & SDEV $(\mathrm{ps})$ \\
\hline 1 & 15.43 & 18 & 497.2 \\
2 & 14.38 & 22 & 478.8 \\
3 & 14.91 & 26 & 443.0 \\
4 & 14.02 & 30 & 412.2 \\
\hline
\end{tabular}




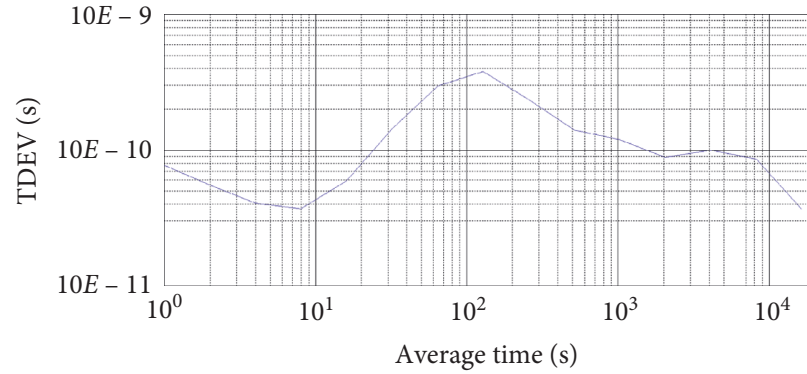

Group 1

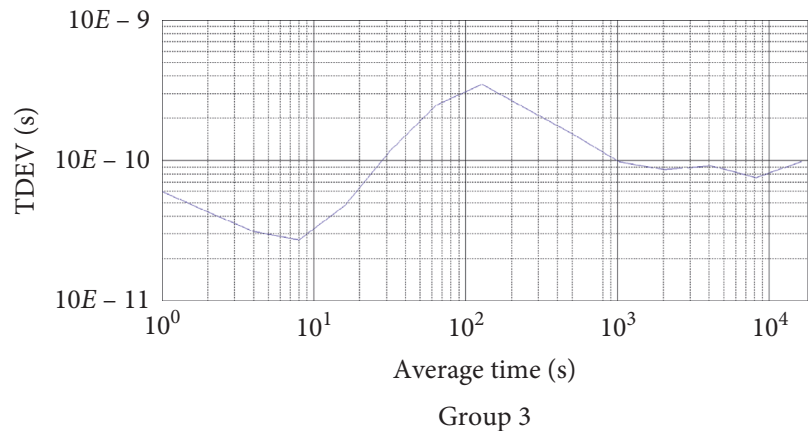

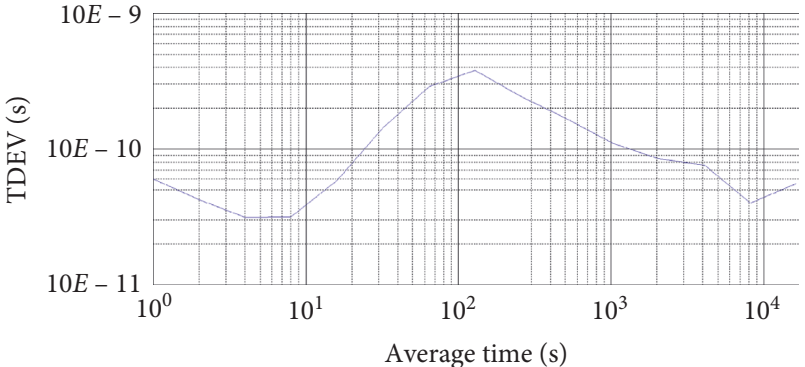

Group 2

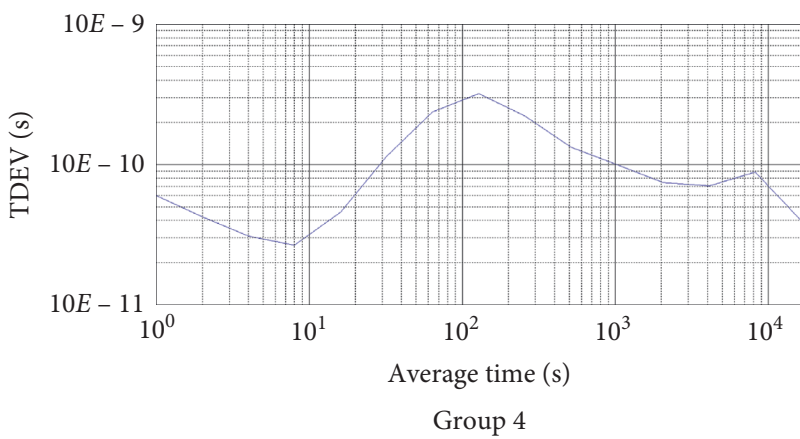

Figure 6: TDEV at two ends of $700 \mathrm{~km}$ optical fiber. (a) Group 1. (b) Group 2. (c) Group 3. (d) Group 4.

TABLE 2: Tdev of four short-term performance tests.

\begin{tabular}{lccc}
\hline Group & Duration $(\mathrm{h})$ & TDEV $(1 \mathrm{~s})(\mathrm{ps})$ & TDEV $(10 \mathrm{E} 4 \mathrm{~s})(\mathrm{ps})$ \\
\hline 1 & 15.43 & 76.64 & $66.91 \mathrm{ps}$ \\
2 & 14.38 & 59.26 & $43.58 \mathrm{ps}$ \\
3 & 14.91 & 59.68 & $80.58 \mathrm{ps}$ \\
4 & 14.02 & 58.84 & $70.35 \mathrm{ps}$ \\
\hline
\end{tabular}
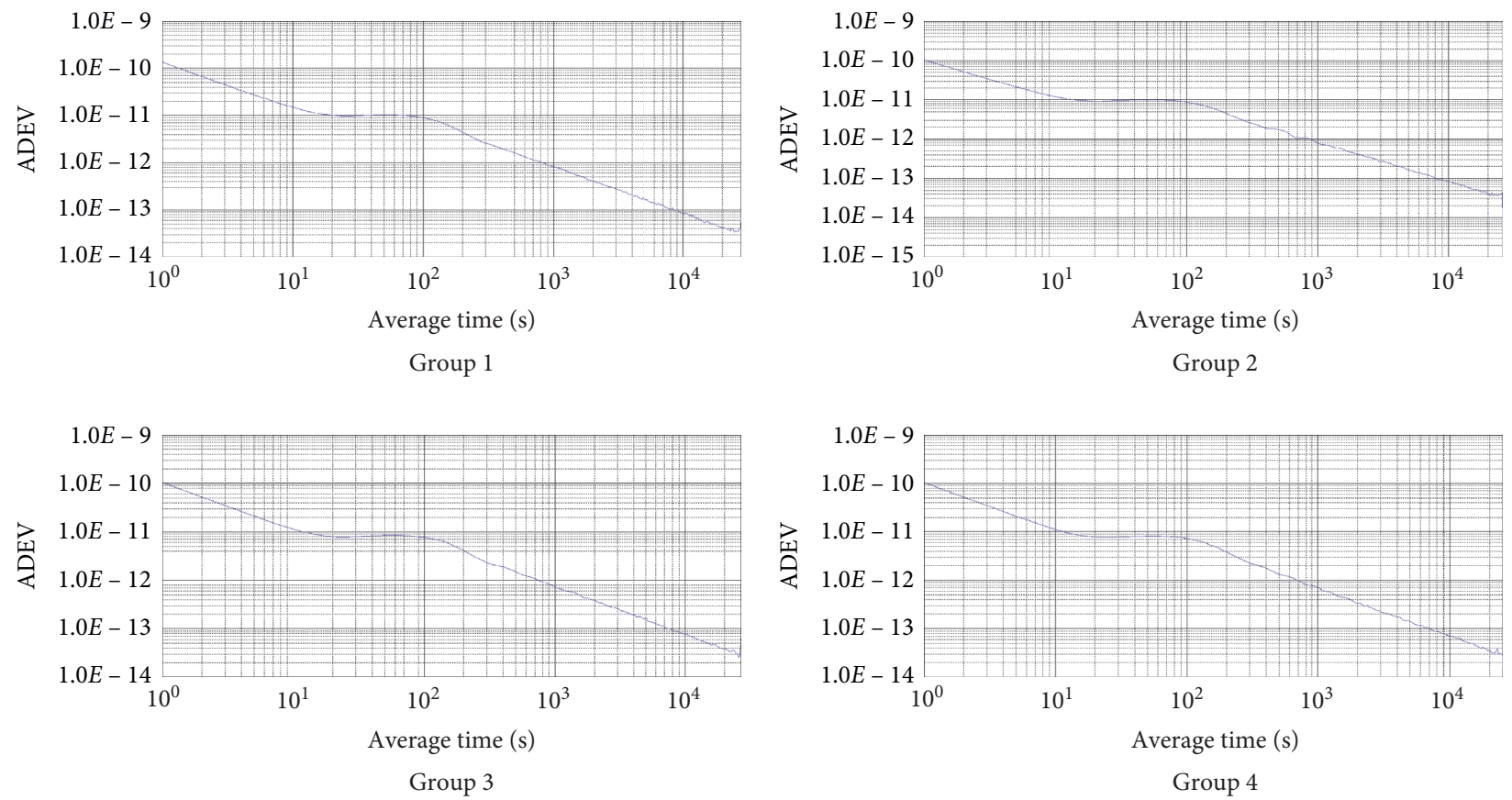

Figure 7: ADEV at two ends of $700 \mathrm{~km}$ optical fiber. (a) Group 1. (b) Group 2. (c) Group 3. (d) Group 4. 
TABle 3: Adev of four short-term performance tests.

\begin{tabular}{lccc}
\hline Group & Duration $(\mathrm{h})$ & ADEV $(1 \mathrm{~s})$ & ADEV $(10 \mathrm{E} 4 \mathrm{~s})$ \\
\hline 1 & 15.43 & $1.3 \times 10^{-10}$ & $8.09 \times 10^{-14}$ \\
2 & 14.38 & $1.02 \times 10^{-10}$ & $7.93 \times 10^{-14}$ \\
3 & 14.91 & $1.045 \times 10^{-10}$ & $7.39 \times 10^{-14}$ \\
4 & 14.02 & $1.04 \times 10^{-10}$ & $7.01 \times 10^{-14}$ \\
\hline
\end{tabular}

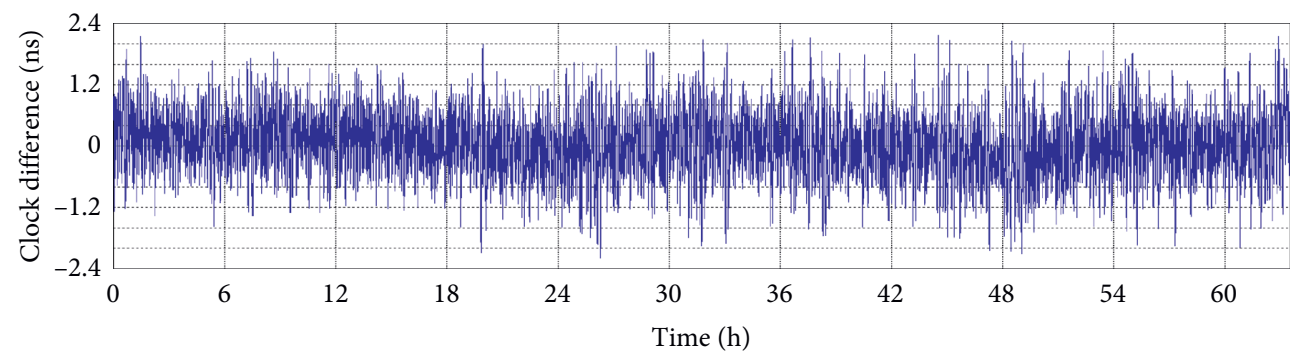

(a)

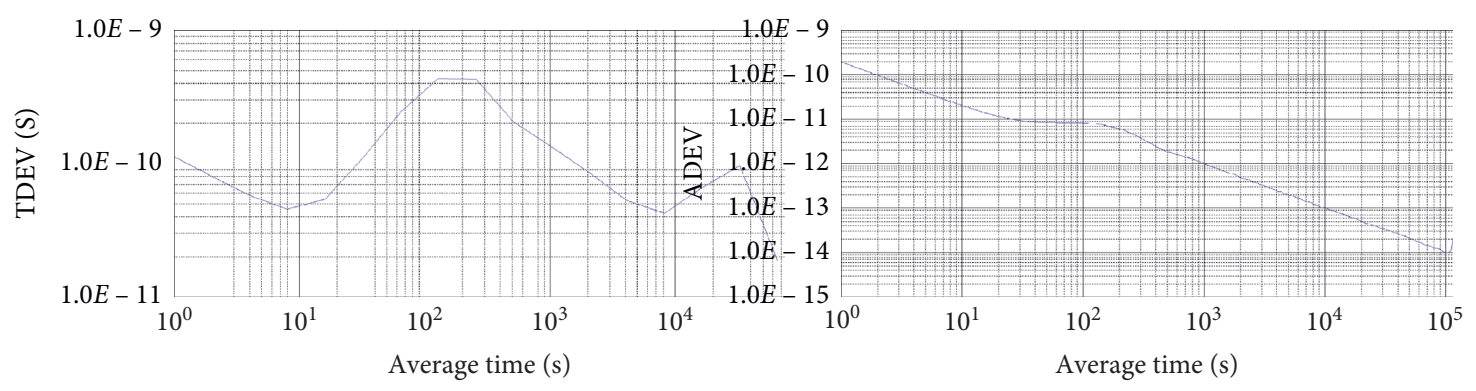

(b)

(c)

FIGURE 8: Long-term measurement results of clock synchronization at two ends of $700 \mathrm{~km}$ optical fiber. (a) Clock difference. (b) TDEV. (c) ADEV.

through $2 \mathrm{~m}$ customized optical fiber. The phase values are compared in the counter, and the data are observed and recorded in real-time.

(4) The experiment was carried out for 55 hours, and the data was recorded.

(5) After the temperature box is started, the internal temperature is set to reduce from $30^{\circ} \mathrm{C}$ to $-20^{\circ} \mathrm{C}$ at the speed of $10^{\circ} \mathrm{C} / \mathrm{h}$ within five hours and then increase from $-20^{\circ} \mathrm{C}$ to $40^{\circ} \mathrm{C}$ at the speed of $20^{\circ} \mathrm{C} / \mathrm{h}$ within three hours.

(6) When the temperature box is shut down, the internal temperature decreases slowly and irregularly.

(7) After the collection of experimental data with a total duration of about 63.5 hours, the equipment is shut down.

The clock difference, time deviation, and Allan deviation data record of the long-term test are shown in Figure 8.

The experimental results show that the system can track the temperature change and adjust the delay compensation value. After the clock difference is calculated, the SDEV of the overall time is $589.8 \mathrm{ps}$, the TDEV is $47.5 \mathrm{ps} / 10^{4} \mathrm{~s}$, and the $\mathrm{ADEV}$ is $1.14 \times 10^{-14} /$ day. The noise sources of the system include the following aspects: (1) the background noise of the end machine of the system under laboratory conditions: the background noise is mainly determined by encoder/decoder, optical transceiver module, and time interval tester. (2) The short-term stability (1-100 s) is mainly related to the signal-to-noise ratio of the received signal. (3) The long-term stability (1000-10000 s) is mainly due to the variation of the transmission delay difference of the optical fiber link with the temperature and the influence of the drift of the transmitting wavelength.

In the field optical fiber, the temperature fluctuation of the fiber core is relatively small, generally less than $5 \%$ of the surface environmental temperature fluctuation, because the fiber core is buried in a certain depth of soil, and the fiber core is protected by the cladding and surface layer. Therefore, the core temperature variation of buried optical cable is generally less than $1^{\circ} \mathrm{C}$. For $100 \mathrm{~km}$ fiber, the time synchronization error caused by $1^{\circ} \mathrm{C}$ temperature fluctuation is about 10 ps. For the nanosecond time synchronization accuracy of the system, the influence of the temperature fluctuation of the field optical fiber is extremely small.

The fluctuation of emission wavelength of master-slave site laser is a factor affecting the system accuracy. In practical engineering, the terminal equipment of each node, including 
rubidium clock, laser, and other hardware equipment, can be placed in the constant temperature and pressure environment, which can make the short-term stability of laser emission wavelength extremely high, and the long-term drift of wavelength can be measured by spectrometer.

This system is a time service solution for multinode optical fiber network. Compared with the traditional optical fiber timing scheme, each scheme has its advantages in network structure and time synchronization accuracy. Besides, the scheme has obvious advantages: 1) each rubidium atomic clock can serve local users and realize multinode download function. (2) In this scheme, low-cost common equipment is used to achieve high time synchronization accuracy. (3) The design is more conducive to the construction of the optical fiber timing network, more in line with the application requirements of optical fiber timing, and has better application potential.

\section{Conclusion}

In conclusion, this paper presents a new time synchronization scheme based on WDM technology and cascade tame clock technology. Each pair of master-slave nodes adopts WDM technology for time synchronization. At the same time, 1 PPS signal, time code signal, and $10 \mathrm{MHz}$ signal are transmitted in one wavelength channel. Time synchronization between master and slave nodes is realized by solving one-way delay value, and high-precision time synchronization between nodes is realized by gradually taming clock. A $700 \mathrm{~km}$ long-distance time-frequency synchronization system is constructed in the laboratory, which is transmitted step by step through eight small rubidium atomic clocks. Through experiments, time synchronization between multiple remote devices and local devices in optical fiber link is realized, which verifies the feasibility of this scheme. On this basis, our next work will use star and ring topology for multisite time synchronization, which will lay a certain foundation for practical and large area network optical fiber time synchronization research.

\section{Data Availability}

The data used to support the findings of this study are available from the corresponding author upon request.

\section{Conflicts of Interest}

The authors declare that they have no conflicts of interest.

\section{Acknowledgments}

This work was supported by the National Key Research and Development Plan under Grant 2016YFB0501700 and Grant 2016 YFB0501701.

\section{References}

[1] J. P. Uzan, "The fundamental constants and their variation: observational and theoretical status," Review of Modern Physics, vol. 75, no. 2, pp. 403-455, 2002.
[2] S. Droste, F. Ozimek, and T. Udem, "Optical-frequency transfer over a single-span $1840 \mathrm{~km}$ fiber link," Physical Review Letters, vol. 111, no. 11, pp. 110801-110805, 2013.

[3] Y. Yang, "Concepts of Comprehensive PNT and related key technologies," Acta Geodaeticaet Cartographica Sinica, vol. 45 , no. 5 , p. 506, 2016.

[4] X. Zhu, B. Wang, and C. Gao, "Fiber-based multiple-access frequency synchronization via $1 \mathrm{f}-2 \mathrm{f}$ dissemination," Chinese Physics B, vol. 25, no. 9, pp. 264-267, 2016.

[5] K.-C. Lin, W.-Y. Lee, and C.-J. Lin, "Effects of gamma radiation on optical fibre sensors," IEE Proceedings - Optoelectronics, vol. 151, no. 1, pp. 12-15, 2004.

[6] T. Akatsuka, H. Ono, and K. Hayashida, "A 30-Km-long optical fiber link for frequency comparison between distant strontium optical lattice clocks," in Proceedings of the European Frequency \& Time Forum and International Frequency Control Symposium, IEEE, Prague, Czech Republic, January 2014.

[7] B. Wang, C. Gao, and W. Chen, "Precise and continuous time and frequency Synchronisation at the $5 \times 10-19$ accuracy level," Scientific Reports, vol. 2, pp. 556-560, 2012.

[8] P. S. Andre, A. N. Pinto, and J. L. Pinto, "Effect of temperature on the single mode fiberschromatic dispersion," Journal of Microwaves and Optoelectronics, vol. 3, no. 5, pp. 64-70, 2004.

[9] A. Czubla, P. Krehlik, and L. Sliwczyliski, "Some approximated methods of calculation Sagnac correction for optical fiber time transfer," in Proceedings of the 2017 Joint Conference of the European Frequency and Time Forum and IEEE International Frequency Control Symposium, pp. 399-401, IEEE, Besancon, France, July 2017.

[10] L. Yu, L. Lu, and R. Wang, "Analysis of the Sagnac effect on the accuracy of the long haul optical fiber time transfer system," in Proceedings of the 2013 Joint European Frequency and Time Forum \&International Frequency Control Symposium, pp. 303-305, Prague, Czech Republic, July 2013.

[11] M. Slapak and J. Vojtech, "Real like transmission lines for Sagnac correction study," in Proceedings of EFTF 2018, Torino, Italy, April 2018.

[12] N. Chiodo, N. Quintin, F. Stefani et al., "Cascaded optical fiber link using the internet network for remote clocks comparison," Optics Express, vol. 23, no. 26, pp. 33927-33937, 2015.

[13] X. Zhu and B. W. Y. Guo, "A robust fiber-based frequency synchronization system immune to dramatic temperature fluctuation," Chinese Optics Letters, vol. 16, no. 1, 2018.

[14] K. Predehl, G. Grosche, S. M. F. Raupach et al., "A 920-kilometer optical fiber link for frequency metrology at the 19th decimal place," Science, vol. 336, no. 6080, pp. 441-444, 2012.

[15] China[Online]. Available: http://www.cas.cn/cm/201910/ t20191012_4720006.shtml.

[16] J. W. L. L. L. Rauch, "The evolution of technology in the deep space network: a history of the advanced systems program," Telecommunications \& Data Acquisition Progress Report, vol. 130, pp. 1-44, 1994.

[17] V. Smotlacha, A. Kuna, and W. Mache, “"Time transfer using fiber links," EFTF-2010 24th European Frequency and Time Forum," IEEE, pp. 1-8, 2010.

[18] P. Krehlik, L. Sliwczynski, L. Buczek, J. Kolodziej, and M. Lipinski, "ELSTAB-fiber-Optic time and frequency distribution technology: a general characterization and fundamental limits," IEEE Transactions on Ultrasonics, Ferroelectrics, and Frequency Control, vol. 63, no. 7, pp. 993-1004, 2016. 
[19] G. Wu and J. Chen, "Ultra-long haul high-precison fiber-optic two way time transfer," Science \& Technology Review, vol. 34, no. 16, pp. 99-103, 2016.

[20] H. Zhang, G. Wu, L. Hu, X. Li, and J. Chen, "High-precision time transfer over 2000-km fiber link," IEEE Photonics Journal, vol. 7, no. 6, pp. 1-9, 2015. 